Canad. J. Math. Vol. 54 (2), 2002 pp. 225-238

\title{
Spaces of Whitney Functions on Cantor-Type Sets
}

\author{
Bora Arslan, Alexander P. Goncharov and Mefharet Kocatepe
}

Abstract. We introduce the concept of logarithmic dimension of a compact set. In terms of this magnitude, the extension property and the diametral dimension of spaces $\mathcal{E}(K)$ can be described for Cantortype compact sets.

\section{Introduction}

We introduce a concept of logarithmic dimension as the following generalization of the Hausdorff dimension: take the function $\psi(r)=\frac{1}{\log \frac{1}{r}}, r>0$, corresponding to the logarithmic measure; then for any compact set $K \subset \mathbb{R}$ there exists a critical value $\lambda_{0}=\lambda_{0}(K) \in[0, \infty]$ (called the logarithmic dimension of $K$ ) such that for $\lambda<\lambda_{0}$ the $\psi^{\lambda}$-measure of $K$ is $\infty$, for $\lambda>\lambda_{0}$ it is zero.

The aim of this article is to show that for the class $\mathcal{E}(K)$ of Whitney functions defined on generalized Cantor sets the logarithmic dimension is highly suitable for the investigation of the following two problems.

The problem of geometric characterization of the extension property of $K$ (that is the existence of a continuous linear extension operator $\left.L: \mathcal{E}(K) \rightarrow C^{\infty}\left(\mathbb{R}^{n}\right)\right)$ was posed by Mityagin [11, Section 8.5]. Some particular results were given by Stein [16], Bierstone [2], Pawłucki and Pleśniak [13] and others. In [17] Tidten suggested to consider perfect sets of class $(\alpha)$ (see [18], [6] for definition) and proved that the condition $K \in(1)$ is sufficient for the extension property of $K$ whereas " $K \in(\alpha)$ for some $\alpha$ " is necessary. In particular he showed that the classical Cantor set $C$ (clearly $\lambda_{0}(C)=\infty$ ) has the extension property. Here we show that the generalized Cantor set $K$ has the extension property if $\lambda_{0}(K)>1$ and it has not if $\lambda_{0}(K)<1$. Examples of perfect sets of finite class without extension property can be given easily (compare this with [6]).

On the other hand, the diametral dimension of the space $\mathcal{E}(K)$ in our case can be described in terms of the logarithmic dimension of $K$ (Chapter 4$)$ and what is more, we have complete (up to the case of equal logarithmic dimensions) isomorphic classification of given spaces $\mathcal{E}(K)$ (Theorem 3 ). Examples of continua of pairwise nonisomorphic spaces of considered type appear directly (compare with [8]). It should be noted that the diametral dimension can not be applied to distinguish nuclear spaces of type $C^{\infty}(\bar{\Omega})$ or $\mathcal{E}(K)$ with $\stackrel{\circ}{K} \neq \varnothing$. In fact, all these spaces contain a subspace which is isomorphic to the space $s$ of rapidly decreasing sequences and thus their diametral dimension $\Gamma(X)$ is not larger than $\Gamma(s)$ (see for example [11, Proposition 7]).

Received by the editors March 6, 2001.

AMS subject classification: 46E10, 31A15, 46A04.

(C) Canadian Mathematical Society 2002. 
On the other hand, the space $s$ has the smallest diametral dimension in the class of nuclear spaces and we get $\Gamma(X)=\Gamma(s)$.

\section{Logarithmic Dimension}

We consider the following generalization of the Cantor ternary set. Let $\left(l_{n}\right)_{1}^{\infty}$ be a sequence of positive numbers and $\left(N_{n}\right)_{1}^{\infty}$ be a sequence of integers, $N_{n} \geq 2$ for all $n$. Then $K=K\left(\left(l_{n}\right),\left(N_{n}\right)\right)=\bigcap_{n=0}^{\infty} E_{n}$, where $E_{0}=I_{0,1}=[0,1]$ and $E_{n}, n \geq 1$, is a union of $N_{1} N_{2} \cdots N_{n}$ disjoint closed intervals $I_{n, k}$ of length $l_{n}$ and $E_{n+1}$ is obtained by replacing each interval by $N_{n+1}$ disjoint subintervals $I_{n+1, j}$ of length $l_{n+1}$ with $N_{n+1}-1$ gaps of length $h_{n+1}$. The intervals $I_{n, k}$ that make up the set $E_{n}$ are called basic intervals. The set $K$ is well-defined if for all $n$ we have $l_{n-1}>N_{n} l_{n}$ with $l_{0}=1$. Then $h_{n}=$ $\frac{l_{n-1}-N_{n} l_{n}}{N_{n}-1}$. We will restrict ourselves to the case $l_{n} \leq h_{n}$, since otherwise $K$ is uniformly perfect and has the extension property.

Let $\alpha_{1}=1$ and for $n \geq 2$ let $\alpha_{n}$ satisfy $l_{n}=l_{n-1}^{\alpha_{n}}$. For $n \geq 2$ set $\lambda_{n}=\frac{\log N_{n}}{\log \alpha_{n}}$. We will analyze two regular cases: $N_{n}=N$ for all $n$ and $N_{n} \nearrow \infty$ as $n \rightarrow \infty$. The corresponding compact set of finite type will be denoted by $K_{N} ; K_{\infty}$ stands for the infinite case. If in particular $\alpha_{n}=\alpha, n \geq 2$, then we will write $K_{N}^{(\alpha)}$ and $K_{\infty}^{(\alpha)}$.

Let us introduce a parameter which can be applied in classifying such rarefied sets as $K_{N}^{(\alpha)}$. Let $\psi(r)=\frac{1}{\log \frac{1}{r}}, r>0$. Here and subsequently, log denotes the natural logarithm, $[x]$ denotes the greatest integer in $x$. For $0<\lambda<\infty$ consider the $\psi^{\lambda}$-measure of a compact set $K$ (see e.g. [12, V.6.2] or [3], [15]): let $m_{\epsilon}\left(K, \psi^{\lambda}\right)=\inf \sum \psi^{\lambda}\left(r_{i}\right)$ where the greatest lower bound is taken over all covers $\bigcup B_{i}$ of $K$ by balls $B_{i}$ with $\operatorname{diam} B_{i}=r_{i} \leq \epsilon$; then $m\left(K, \psi^{\lambda}\right)=\lim _{\epsilon \rightarrow 0} m_{\epsilon}\left(K, \psi^{\lambda}\right)$. As in the definition of the Hausdorff dimension (see e.g. $[15,10.1],[3,1.2]$ ) we see that there exists a critical value $\lambda_{0}=\lambda_{0}(K), 0 \leq \lambda_{0} \leq \infty$, such that $m\left(K, \psi^{\lambda}\right)=\infty$ for $\lambda<\lambda_{0}$ and $m\left(K, \psi^{\lambda}\right)=0$ for $\lambda>\lambda_{0}$. Since the function $\psi$ corresponds to the logarithmic measure we will say that the value $\lambda_{0}$ is the logarithmic dimension of $K$.

Proposition 1 Suppose that for $K=K\left(\left(l_{n}\right),\left(N_{n}\right)\right)$ the limit $\lambda_{0}=\lim _{n} \lambda_{n}$ exists in the set of extended real numbers. Then $\lambda_{0}$ is the logarithmic dimension of $K$. In particular, $\lambda_{0}\left(K_{N}^{(\alpha)}\right)=\frac{\log N}{\log \alpha}$.

Proof For $\lambda>\lambda_{0}$ consider $m_{\epsilon}\left(K, \psi^{\lambda}\right)$. Notice that $\alpha_{n} \nrightarrow 1$. In fact, otherwise we would have $\lambda_{0}=\infty$. For $\epsilon=l_{n}$ we have the covering of $K$ by $N_{1} N_{2} \cdots N_{n}$ intervals $I_{n, k}$ of length $\epsilon$. Thus

$$
m_{\epsilon}\left(K, \psi^{\lambda}\right) \leq N_{1} N_{2} \cdots N_{n} \log ^{-\lambda}\left(\frac{1}{l_{n}}\right) .
$$

Since $l_{n}=l_{1}^{\alpha_{1} \alpha_{2} \cdots \alpha_{n}}$ and $N_{n}=\alpha_{n}^{\lambda_{n}}$ we get

$$
m_{\epsilon}\left(K, \psi^{\lambda}\right) \leq \alpha_{1}^{\lambda_{1}-\lambda} \cdots \alpha_{n}^{\lambda_{n}-\lambda} \log ^{-\lambda}\left(\frac{1}{l_{1}}\right),
$$

which tends to 0 as $n \rightarrow \infty$ since $\lambda>\lim \lambda_{n}$ and $\alpha_{n} \nrightarrow 1$. Thus, $m\left(K, \psi^{\lambda}\right)=0$. 
Suppose now that $\lambda<\lambda_{0}$. Then we can modify the arguments in [3,2.3]. For $\epsilon>$ 0 there exists a finite covering $\bigcup_{i=1}^{M} U_{i}$ of $K$ by open intervals $U_{i}$, diam $U_{i}=r_{i}<2 \epsilon$ such that $\sum \psi^{\lambda}\left(r_{i}\right) \leq 2 m_{\epsilon}\left(K, \psi^{\lambda}\right)$. For each $r_{i}$ fix $n=n(i) \in \mathbb{N}$ with $l_{n} \leq r_{i}<l_{n-1}$. Let $n_{0}=\min _{i \leq M} n(i), n_{1}=\max _{i \leq M} n(i)$. To simplify calculations we set $l_{1}=1 / e$. Then

$$
\psi^{\lambda}\left(r_{i}\right) \geq \psi^{\lambda}\left(l_{n}\right) \geq\left(\alpha_{1} \cdots \alpha_{n}\right)^{-\lambda} .
$$

Let $\epsilon$ be so small that $\lambda_{n}>\lambda$ for $n \geq n_{0}$. Then

$$
\begin{aligned}
\left(\alpha_{1} \cdots \alpha_{n}\right)^{\lambda} & =\left(\alpha_{1} \cdots \alpha_{n_{0}-1}\right)^{\lambda} \cdot\left(\alpha_{n_{0}}^{\lambda} \cdots \alpha_{n}^{\lambda}\right) \\
& \leq\left(\alpha_{1} \cdots \alpha_{n_{0}-1}\right)^{\lambda} \cdot\left(\alpha_{n_{0}}^{\lambda_{n_{0}}} \cdots \alpha_{n}^{\lambda_{n}}\right) \\
& =\left(\alpha_{1} \cdots \alpha_{n_{0}-1}\right)^{\lambda} \cdot N_{n_{0}} \cdots N_{n} .
\end{aligned}
$$

We decompose the sum $\sum \psi^{\lambda}\left(r_{i}\right)$ into two parts. Let $\sum^{\prime}$ be the sum over all $i$ such that $l_{n} \leq r_{i}<\frac{l_{n-1}}{N_{n}}$, and $\sum^{\prime \prime}$ be the sum over the remaining $i$ 's. Since $\frac{l_{n-1}}{N_{n}}<l_{n}+h_{n}$, for any $i$ in the sum $\sum^{\prime}$, the interval $U_{i}$ can intersect at most two basic intervals of $E_{n}$. By construction, it can intersect at most $2 N_{n+1}$ basic intervals of $E_{n+1} ; \cdots ; 2 N_{n+1} \cdots N_{n_{1}}$ basic intervals of $E_{n_{1}}$. Then

$$
\begin{aligned}
2 N_{n+1} \cdots N_{n_{1}} & \leq 2 N_{n+1} \cdots N_{n_{1}} \cdot\left(\alpha_{1} \cdots \alpha_{n}\right)^{\lambda} \cdot \psi^{\lambda}\left(r_{i}\right) \\
& \leq 2 N_{n_{0}} \cdots N_{n_{1}} \cdot\left(\alpha_{1} \cdots \alpha_{n_{0}-1}\right)^{\lambda} \cdot \psi^{\lambda}\left(r_{i}\right)
\end{aligned}
$$

For $i$ in the second sum $\sum^{\prime \prime}$, fix $j, j=1,2, \ldots, N_{n}-1$, such that $\frac{j}{N_{n}} l_{n-1} \leq r_{i}<$ $\frac{j+1}{N_{n}} l_{n-1}$. Then the interval $U_{i}$ can intersect at most $j+2$ basic intervals of $E_{n}$ and thus $(j+2) N_{n+1} \cdots N_{n_{1}}$ basic intervals of $E_{n_{1}}$. Here

$$
\psi^{\lambda}\left(r_{i}\right) \geq \psi^{\lambda}\left(\frac{j}{N_{n}} l_{n-1}\right) \geq\left(\alpha_{1} \cdots \alpha_{n-1}+\log \frac{N_{n}}{j}\right)^{-\lambda}
$$

If $\log \frac{N_{n}}{j} \geq \alpha_{1} \cdots \alpha_{n-1}$, then $1 \leq 2^{\lambda} \log ^{\lambda}\left(\frac{N_{n}}{j}\right) \psi^{\lambda}\left(r_{i}\right) \leq C_{\lambda}^{\prime} \frac{N_{n}}{j} \psi^{\lambda}\left(r_{i}\right)$. Therefore

$$
\begin{aligned}
(j+2) N_{n+1} \cdots N_{n_{1}} & \leq C_{\lambda}^{\prime \prime} N_{n} N_{n+1} \cdots N_{n_{1}} \psi^{\lambda}\left(r_{i}\right) \\
& \leq C_{\lambda}^{\prime \prime} N_{n_{0}} \cdots N_{n_{1}} \cdot\left(\alpha_{1} \cdots \alpha_{n_{0}-1}\right)^{\lambda} \psi^{\lambda}\left(r_{i}\right)
\end{aligned}
$$

On the other hand, if $\log \frac{N_{n}}{j}<\alpha_{1} \cdots \alpha_{n-1}$, then $1 \leq 2^{\lambda}\left(\alpha_{1} \cdots \alpha_{n-1}\right)^{\lambda} \psi^{\lambda}\left(r_{i}\right)$, therefore

$$
\begin{aligned}
(j+2) N_{n+1} \cdots N_{n_{1}} & \leq\left(N_{n}+1\right) N_{n+1} \cdots N_{n_{1}}\left(\alpha_{1} \cdots \alpha_{n-1}\right)^{\lambda} 2^{\lambda} \psi^{\lambda}\left(r_{i}\right) \\
& \leq 2^{\lambda+1} N_{n_{0}} \cdots N_{n_{1}}\left(\alpha_{1} \cdots \alpha_{n_{0}-1}\right)^{\lambda} \psi^{\lambda}\left(r_{i}\right)
\end{aligned}
$$

Thus any interval $U_{i}, i \leq M$ can intersect at most

$$
C_{\lambda} N_{n_{0}} \cdots N_{n_{1}}\left(\alpha_{1} \cdots \alpha_{n_{0}-1}\right)^{\lambda} \psi^{\lambda}\left(r_{i}\right)
$$


basic intervals of $E_{n_{1}}$. Here $C_{\lambda}=\max \left\{C_{\lambda}^{\prime \prime}, 2^{\lambda+1}\right\}$. Since the covering $\bigcup U_{i}$ intersects all basic intervals of $E_{n_{1}}$, we have

$$
N_{1} \cdots N_{n_{1}} \leq C_{\lambda} N_{n_{0}} \cdots N_{n_{1}}\left(\alpha_{1} \cdots \alpha_{n_{0}-1}\right)^{\lambda} \sum \psi^{\lambda}\left(r_{i}\right)
$$

and so

$$
\sum \psi^{\lambda}\left(r_{i}\right) \geq C_{\lambda}^{-1} N_{1} \alpha_{2}^{\lambda_{2}-\lambda} \cdots \alpha_{n_{0}-1}^{\lambda_{n_{0}-1}-\lambda} .
$$

This bound implies that the sum of type $\sum \psi^{\lambda}\left(r_{i}\right)$ must be arbitrarily large for small enough $\epsilon$, that is $m\left(K, \psi^{\lambda}\right)=\infty$.

In fact, for $\alpha_{n} \nrightarrow 1$ it is obvious as $\lambda_{n}>\lambda$ for large $n$. Clearly, $\epsilon \rightarrow 0$ gives $n_{0} \rightarrow \infty$. If $\alpha_{n} \rightarrow 1$, then $\alpha_{n}^{\lambda_{n}-\lambda}=\frac{N_{n}}{\alpha_{n}^{\lambda}} \geq \frac{2}{\alpha_{n}^{\lambda}} \rightarrow 2$ as $n \rightarrow \infty$ and the result follows.

The value $\lambda_{0}=1$ is critical in Potential Theory: if $\lambda_{0}(K)<1$, then the logarithmic measure of $K$ is 0 and the set $K$ is exceptional. We show that this bound is crucial as well for the extension property of Cantor-type sets.

\section{Extension Property}

Let $\mathcal{E}(K)$ denote the space of Whitney functions on a perfect compact set $K$ with the topology defined by the norms

$\|f\|_{q}=|f|_{q}+\sup \left\{\left|\left(R_{y}^{q} f\right)^{(j)}(x)\right| \cdot|x-y|^{j-q}: x, y \in K, x \neq y, j \leq q\right\}, \quad q=0,1, \ldots$,

where $|f|_{q}=\sup \left\{\left|f^{(j)}(x)\right|: x \in K, j \leq q\right\}$ and $R_{y}^{q} f(x)=f(x)-T_{y}^{q} f(x)$ is the Taylor remainder. Each function $f \in \mathcal{E}(K)$ is extendable to a $C^{\infty}$-function on the line. If there exists a linear continuous extension operator $L: \mathcal{E}(K) \rightarrow C^{\infty}(\mathbb{R})$, then we say that the compact set $K$ has the extension property. In [17] Tidten showed that the extension property of $K$ and the property DN of the space $\mathcal{E}(K)$ are equivalent.

A Fréchet space $X$ with a fundamental system of seminorms $\left(\|\cdot\|_{q}\right)$ is said to have the property DN [21] (see also the class $D_{1}$ in [23]) if

$$
\exists p \forall q \exists r, C>0:\|\cdot\|_{q} \leq t\|\cdot\|_{p}+\frac{C}{t}\|\cdot\|_{r}, \quad t>0 .
$$

Here $p, q, r \in \mathbb{N}_{0}=\{0,1,2, \ldots\}$.

Proposition 2 For $X=\mathcal{E}(K)$, the following statements are equivalent to $\mathrm{DN}$ :

(a) $\exists p \exists R>0 \forall q \exists r, C:|\cdot|_{q} \leq t^{R \cdot q}\|\cdot\|_{p}+\frac{C}{t^{q}}\|\cdot\|_{r}, t>0$;

(b) $\exists p \forall \epsilon>0 \forall q \exists r, C:\left.|\cdot|\right|_{q} ^{1+\epsilon} \leq C\|\cdot\|_{p}\|\cdot\|_{r}^{\epsilon}$.

Proof In [10, Lemma 29.10] and in [5] it was shown that DN is equivalent to the conditions (a), (b) with $\|\cdot\|_{q}$ instead of $|\cdot|_{q}$. Frerick [4] and Tidten [19] proved that we can replace $\|\cdot\|_{q}$ by $|\cdot|_{q}$.

We first generalize Theorems 2 and 3 in [6].

Theorem 1 If $\left\lfloor\right.$ lim $\alpha_{n}>N$, then $K_{N}$ does not have the extension property. If $\overline{\lim } \alpha_{n}<$ $N$, then $K_{N}$ has the extension property. 
Proof Let $\beta$ be such that $\underline{\lim } \alpha_{n}>\beta>N$ and find $i_{0}$ such that $\alpha_{i}>\beta, i \geq i_{0}$. Fix $0<\epsilon<\frac{\beta-N}{2(N-1)}$ and $M \in \mathbb{N}$ such that $M \geq \frac{2 \beta}{\beta-N}$. We want to show

$$
\forall p \exists \epsilon \exists q: \forall r>q \exists\left(f_{n}\right) \in \mathcal{E}\left(K_{N}\right): \frac{\left\|f_{n}\right\|_{p} \cdot\left\|f_{n}\right\|_{r}^{\epsilon}}{\left|f_{n}\right|_{q}^{1+\epsilon}} \rightarrow 0 \quad \text { as } \quad n \rightarrow \infty
$$

For arbitrary $p \in \mathbb{N}$ let $q=M p$. For any $r>q$ take $s \in \mathbb{N}$ with $N^{s} \geq \frac{r}{q}>N^{s-1}$. Fix a natural number $n, n \geq s+i_{0}$ and consider first $N^{s}$ intervals of $E_{n}$. Let $c_{j}$ denote the midpoint of $I_{n, j}, j=1,2, \ldots, N^{s}$. Set $f_{n}(x)=g^{q}(x)$ where $g(x)=\Pi_{j=1}^{N^{s}}\left(x-c_{j}\right)$ for $x \in K_{N} \cap\left[0, l_{n-s}\right]$ and $g(x)=0$ otherwise on $K_{N}$. Let us estimate the norms of $f_{n}$.

Fix $k \in \mathbb{N}, k \leq p$ and $x \in \bigcup_{j=1}^{N^{s}} I_{n, j}$. By Lemma 1 in [6], we have

$$
\left|f_{n}^{(k)}(x)\right| \leq C_{p, r}|g(x)|^{q-k}
$$

where $C_{p, r}=\frac{\left(N^{s} \cdot q\right) !}{\left(N^{s} \cdot q-k\right) !}<(N \cdot r)^{p}$ does not depend on $n$.

By the structure of $K_{N}$ we have $|g(x)|<l_{n} \tau^{N-1}$ where $\tau=l_{n-1} l_{n-2}^{N} \cdots l_{n-s}^{N^{s-1}}$. Thus

$$
\left|f_{n}\right|_{p} \leq C_{p, r}\left(l_{n} \tau^{N-1}\right)^{q-p}
$$

Next we estimate

$$
A_{p}:=\frac{\left|\left(R_{x}^{p} f_{n}\right)^{(k)}(y)\right|}{|x-y|^{p-k}}, \quad k \leq p, x \neq y, x, y \in K_{N} .
$$

If $|x-y|<h_{n}$, then $x, y$ belong to the same $I_{n, j}$ for some $j$. Hence applying the Lagrangian form for Taylor's remainder we find $\xi \in I_{n, j}$ such that

$$
\left(R_{x}^{p} f_{n}\right)^{(k)}(y)=\left[f^{(p)}(\xi)-f^{(p)}(x)\right] \frac{(y-x)^{p-k}}{(p-k) !}
$$

and so $A_{p} \leq 2 C_{p, r}\left(l_{n} \tau^{N-1}\right)^{q-p}$.

If $|x-y| \geq h_{n}$, then by (1), (2)

$$
\begin{aligned}
A_{p} & \leq\left|f_{n}^{(k)}(y)\right| \cdot|x-y|^{k-p}+\sum_{i=k}^{p}\left|f_{n}^{(i)}(x)\right| \cdot \frac{|x-y|^{i-p}}{(i-k) !} \\
& \leq C_{p, r}\left(l_{n} \cdot \tau^{N-1}\right)^{q-p}\left[\left(\frac{l_{n} \cdot \tau^{N-1}}{h_{n}}\right)^{p-k}+\sum_{i=k}^{p} \frac{1}{(i-k) !}\left(\frac{l_{n} \cdot \tau^{N-1}}{h_{n}}\right)^{p-i}\right] .
\end{aligned}
$$

Since $l_{n} \cdot \tau^{N-1}<h_{n}$ we get

$$
A_{p} \leq C_{p, r}(1+e)\left(l_{n} \cdot \tau^{N-1}\right)^{q-p} \quad \text { and } \quad\|f\|_{p} \leq C_{p, r}(2+e)\left(l_{n} \cdot \tau^{N-1}\right)^{q-p} .
$$


Clearly

$$
\begin{gathered}
\left|f_{n}\right|_{q} \geq\left|f^{(q)}\left(c_{1}\right)\right|=q !\left|g^{\prime}\left(c_{1}\right)\right|^{q} \\
\left|g^{\prime}\left(c_{1}\right)\right|=\Pi_{j=2}^{N^{s}}\left(c_{j}-c_{1}\right)>\left(\frac{l_{n-1}}{N}\right)^{N-1} \cdot\left(\frac{l_{n-2}}{N}\right)^{N^{2}-N} \cdots\left(\frac{l_{n-s}}{N}\right)^{N^{s}-N^{s-1}} \\
=\tau^{N-1} \cdot N^{1-N^{s}} .
\end{gathered}
$$

Thus,

$$
\left|f_{n}\right|_{q} \geq q ! N^{-r \cdot N} \cdot \tau^{(N-1) q}
$$

Analogously, $\left\|f_{n}\right\|_{r} \leq C_{r}$.

Finally, we conclude for some $D$ independent of $n$ that

$$
\begin{aligned}
\frac{\left\|f_{n}\right\|_{p}\left\|f_{n}\right\|_{r}^{\epsilon}}{\left|f_{n}\right|_{q}^{1+\epsilon}} & \leq D l_{n}^{q-p} \tau^{-(N-1)(\epsilon q+p)} \\
& =D l_{n-s}^{\left(\alpha_{n} \cdots \alpha_{n-s+1}\right)(q-p)}\left[l_{n-s}^{\alpha_{n-1} \cdots \alpha_{n-s+1}} l_{n-s}^{\left(\alpha_{n-2} \cdots \alpha_{n-s+1}\right) N} \cdots l_{n-s}^{N^{s-1}}\right]^{-(N-1)(\epsilon q+p)} .
\end{aligned}
$$

Let us now show that

$$
\begin{aligned}
w:= & \left(\alpha_{n} \cdots \alpha_{n-s+1}\right)(q-p) \\
& \quad-(N-1)(\epsilon q+p)\left[\left(\alpha_{n-1} \cdots \alpha_{n-s+1}\right)+N\left(\alpha_{n-2} \cdots \alpha_{n-s+1}\right)+\cdots+N^{s-1}\right]
\end{aligned}
$$

which is the exponent of $l_{n-s}$, is positive and bounded away from zero. Indeed for the expression in the square brackets we have

$$
[\cdots] \leq \alpha_{n-1} \cdots \alpha_{n-s+1} \cdot \frac{\beta}{\beta-N} \leq \alpha_{n} \cdots \alpha_{n-s+1} \cdot \frac{1}{\beta-N}=: w_{1},
$$

therefore

$$
\begin{aligned}
w & \geq w_{1} \cdot[(\beta-N)(q-p)-(N-1)(\epsilon q+p)] \\
& =w_{1} \cdot p \cdot\{M[\beta-N-\epsilon(N-1)]-\beta+1\} \geq w_{1} \cdot p
\end{aligned}
$$

due to the choice of $M$ and $\epsilon$.

So $w \geq \beta^{s} \frac{p}{\beta-N}$ from which it follows that

$$
\lim _{n \rightarrow \infty} \frac{\left\|f_{n}\right\|_{p}\left\|f_{n}\right\|_{r}^{\epsilon}}{\left|f_{n}\right|_{q}^{1+\epsilon}}=0
$$

Therefore the condition (b) is not fulfilled and the compact set $K_{N}$ does not have the extension property.

The second statement of the theorem can be proved quite similarly to Theorem 3 in [6], so we omit it.

Corollary For a compact set $K_{N}$, let the limit $\alpha=\lim \alpha_{n}$ exist and be not equal to $N$. Then $K_{N}$ has the extension property if and only if $\lambda_{0}\left(K_{N}\right)>1$. 
Remark The set $K_{N}^{(\alpha)}, \alpha>N$, gives us an example of a perfect set of finite class without extension property (for the definition and details see [18], [6]).

We now turn to the case $N_{n} \nearrow \infty$. The compact set $K_{\infty}$ loses its Cantor-type set nature in the following sense: topologically the space $\mathcal{E}\left(K_{\infty}\right)$ is closer to the space $\mathcal{E}(K)$ with $K$ having the form of a sequence of intervals tending to a point than to $\mathcal{E}\left(K_{N}\right)$. Compare the next statement with Theorem 2 in [7].

Theorem 2 The set $K_{\infty}$ has the extension property if and only if there exists a constant $M$ such that

$$
l_{n} \geq h_{n}^{M}, \quad \forall n
$$

Proof Assume the space $\mathcal{E}\left(K_{\infty}\right)$ has the property DN. Let us fix $p$ in the condition (b) of Proposition 2. For $\epsilon=1$ and $q=p+1$ we find $r$ and $C$ such that (b) is fulfilled.

Defining

$$
f_{n}(x)= \begin{cases}\frac{x^{q}}{q !} & \text { if } x \in K \cap\left[0, l_{n}\right] \\ 0 & \text { otherwise }\end{cases}
$$

we obtain the estimates

$$
\left|f_{n}\right|_{q} \geq 1, \quad\left\|f_{n}\right\|_{p} \leq 4 l_{n}, \quad\left\|f_{n}\right\|_{r} \leq 4 h_{n}^{q-r}
$$

Now, Proposition 2 (b) gives $1 \leq 16 C \cdot l_{n} \cdot h_{n}^{q-r}$, from which the necessity follows.

In order to prove the sufficiency we use a simplified version of Lemma 2 in [7] (see also [8]):

Lemma Let $K \subset \mathbb{R}$ be a compact set containing $r+1$ distinct points $x_{0}, x_{1}, \ldots, x_{r}$ such that for some $h$ and a constant $N$

$$
h \leq\left|x_{i}-x_{j}\right| \leq N \cdot h, \quad i, j=0,1, \ldots, r, \quad i \neq j .
$$

Then for all $k \leq r$ and $f \in \mathcal{E}(K)$,

$$
\left|f^{(k)}\left(x_{0}\right)\right| \leq C \cdot h^{-k}|f|_{0}+C \cdot h^{r-k}\|f\|_{r},
$$

where $C$ depends only on $r$ and $N$.

We will show that

$$
\exists p \forall q \exists r, C:|\cdot|_{q} \leq C t^{M q} \cdot\|\cdot\|_{p}+\frac{C}{t^{q}}\|\cdot\|_{r}, \quad t>0,
$$

which is equivalent to (a) and hence to the property DN. The constant $M$ here is the same as the one in the statement of the theorem.

Let $p=0$. Given $q$, let $r=2 q$. Let $n_{0}$ be such that $\frac{N_{n}}{2} \geq r$ for $n \geq n_{0}$. Let $t_{0}=1 / l_{n_{0}-1}$. Given $t \geq t_{0}$, find $n \geq n_{0}$ such that

$$
l_{n}<\frac{1}{t} \leq l_{n-1}
$$


Let $x_{0} \in K$. Then $x_{0} \in I_{n, j_{0}}$ where without loss of generality we may assume that $1 \leq j_{0} \leq N_{n}$. Let $a=a_{n, r+1}$ be the left endpoint of $I_{n, r+1}$. Suppose that $j_{0} \leq \frac{N_{n}}{2}$ and consider the interval $\left[x_{0}, x_{0}+\frac{1}{2 t}\right]$. (If $j_{0}>\frac{N_{n}}{2}$, then similar arguments apply to $\left[x_{0}-\frac{1}{2 t}, x_{0}\right]$.)

Case 1: $\frac{1}{2 t} \geq a$. Let $J$ be such that the interval $\left[x_{0}, x_{0}+\frac{1}{2 t}\right]$ intersects $J+1$ basic intervals of the set $E_{n}$. Then $J \geq r$ due to the choice of $t$ and $\frac{J}{2}\left(l_{n}+h_{n}\right)<\frac{1}{2 t}<$ $2 J\left(l_{n}+h_{n}\right)$, as is easy to check. Then $S:=[J / r] \geq 1$, the points $x_{i}=x_{0}+i S\left(l_{n}+h_{n}\right)$, $i=1, \ldots, r$, belong to $K$ and for $i \neq j,\left|x_{i}-x_{j}\right| \geq S\left(l_{n}+h_{n}\right)>\frac{1}{8 r t}$ since $\left[\frac{J}{r}\right]>\frac{J}{2 r}$ for $J \geq r$. On the other hand, $\left|x_{i}-x_{j}\right| \leq r S\left(l_{n}+h_{n}\right)<\frac{1}{t}$, so by the Lemma

$$
\left|f^{(k)}\left(x_{0}\right)\right| \leq C \cdot t^{k}|f|_{0}+C \cdot t^{k-r}\|f\|_{r} .
$$

Case 2: $\frac{1}{2 t}<a$. Now we choose the points $x_{i}, i=1, \ldots, r$ in the interval $I_{n, j_{0}}$ in a similar way such that $\frac{l_{n}}{4 r} \leq\left|x_{i}-x_{j}\right| \leq \frac{l_{n}}{2}$ for $i \neq j$. Since $a=r\left(l_{n}+h_{n}\right) \leq 2 r h_{n} \leq$ $2 r \cdot l_{n}^{\frac{1}{M}}$, by the condition, we can apply the Lemma with $h=\frac{l_{n}}{4 r}>(4 r)^{-M-1} \cdot t^{-M}$ and $h<(4 r t)^{-1}$ :

$$
\left|f^{(k)}\left(x_{0}\right)\right| \leq C \cdot t^{k \cdot M}|f|_{0}+C \cdot t^{k-r}\|f\|_{r} .
$$

We have this estimate for any $x_{0} \in K$ and $k \leq q$, thus (3) is proved.

We observe that the logarithmic dimension is not related to the extension property of compact sets $K_{\infty}$ of infinite type as seen by the following proposition and example. But in the finite case we can use it as well for isomorphic classification of corresponding spaces.

Proposition 3 If $K_{\infty}$ has the extension property, then $\lambda_{0}\left(K_{\infty}\right)=\infty$.

Proof If $\overline{\lim } \alpha_{n}<\infty$, then clearly $\lim _{n} \lambda_{n}=\infty$. So we consider the case of $\overline{\lim } \alpha_{n}=$ $\infty$. By the previous theorem there is $M \geq 1$ such that $l_{n} \geq h_{n}^{M}$ for all $n$. Let $I=\{n \in$ $\left.\mathbb{N}: \alpha_{n} \geq 2 M\right\}, J=\mathbb{N} \backslash I$. Then $\lim _{n \in J} \lambda_{n}=\infty$. For $n \in I$,

$$
\begin{aligned}
l_{n}^{1 / \alpha_{n}} & =l_{n-1} \leq 2 N_{n} h_{n} \leq 2 N_{n} l_{n}^{1 / M} \Rightarrow \frac{1}{2} l_{n}^{\frac{1}{\alpha_{n}}-\frac{1}{M}} \leq N_{n} \Rightarrow \log N_{n} \\
& \geq \frac{1}{2}\left(\frac{1}{M}-\frac{1}{\alpha_{n}}\right) \log \left(\frac{1}{l_{n}}\right) \geq \frac{1}{4 M} \alpha_{n} \cdots \alpha_{2} \log \left(\frac{1}{l_{1}}\right) \Rightarrow \lambda_{n} \\
& =\frac{\log N_{n}}{\log \alpha_{n}} \geq \frac{\log \left(l_{1}^{-1}\right)}{4 M} \cdot \frac{\alpha_{n} \cdots \alpha_{2}}{\log \alpha_{n}} \rightarrow \infty \quad \text { as } n \rightarrow \infty .
\end{aligned}
$$

But the converse of the above proposition is not true as the following example shows:

Example 1 Let $l_{n}=\exp (-n !)$ and $N_{n}=\left[n^{\log n}+1\right]$. Then $\alpha_{n}=n$ and $\lambda_{n} \geq \log n$. Thus $\lambda_{0}=\infty$. But

$$
h_{n}>\frac{l_{n-1}}{e \cdot\left(N_{n}-1\right)} \geq \frac{1}{n^{\log n} e^{(n-1) !+1}}>\frac{1}{e^{\epsilon \cdot n !}}=l_{n}^{\epsilon}, \quad n \geq n_{0}
$$

for every $\epsilon>0$ which means that $K_{\infty}$ does not have the extension property. 


\section{Diametral Dimension of $\mathcal{E}(K)$}

Approximative and diametral dimensions, introduced by Kolmogorov [9], Pełczyński [14] and Bessaga, Pełczyński and Rolewicz [1], were the first linear topological invariants applicable to isomorphic classification of nonnormed Fréchet spaces. We follow the notation of [11].

Let $X$ be a Fréchet space with a fundamental system of neighborhoods $\left(U_{q}\right)$, let $d_{n}\left(U_{q}, U_{p}\right)$ denote the $n$-th Kolmogorov diameter of $U_{q}$ with respect to $U_{p}$. Then

$$
\Gamma(X)=\left\{\left(\gamma_{n}\right)_{n=0}^{\infty}: \forall p \exists q: \gamma_{n} \cdot d_{n}\left(U_{q}, U_{p}\right) \rightarrow 0 \text { as } n \rightarrow \infty\right\} .
$$

We will consider the counting function corresponding to the diametral dimension

$$
\beta(t)=\beta\left(U_{p}, U_{q}, t\right)=\min \left\{\operatorname{dim} L: t \cdot U_{q} \subset U_{p}+L\right\}, \quad t>0 .
$$

It is easy to see that $\beta(t)=\left|\left\{n: d_{n}\left(U_{q}, U_{p}\right)>\frac{1}{t}\right\}\right|$, where $|Z|$ denotes the cardinality of the set $Z$. If $X$ is a Schwartz space and $p, q$ are sufficiently apart, then the function $\beta$ takes finite values. Clearly, the diametral dimension can be characterized in terms of $\beta$ in the following way.

Proposition $4\left(\gamma_{n}\right) \in \Gamma(X) \Leftrightarrow \forall p \exists q: \forall C \exists n_{0}: \beta\left(U_{p}, U_{q}, C \gamma_{n}\right) \leq n$ for $n \geq n_{0}$.

But we will directly compare asymptotic behavior of counting functions of isomorphic spaces. The proof of the following proposition is straightforward.

Proposition 5 If Fréchet spaces $X$ and $Y$ are isomorphic, then

$$
\forall p_{1} \exists p \forall q \exists q_{1}, \epsilon>0: \beta_{Y}\left(V_{p_{1}}, V_{q_{1}}, \epsilon t\right) \leq \beta_{X}\left(U_{p}, U_{q}, t\right), \quad t>0 .
$$

An analogous condition holds after interchanging $\beta_{X}$ and $\beta_{Y}$. Here $\left(V_{k}\right)_{0}^{\infty}$ is a fundamental system of neighborhoods of $Y$.

Theorem 3 Let $X=\mathcal{E}(K)$ with $K=K\left(\left(l_{n}\right),\left(N_{n}\right)\right)$, let $p$ and $q, p<q$ be fixed natural numbers. If $t \leq \frac{1}{5} l_{n}^{p-q}$, then $\beta\left(U_{p}, U_{q}, t\right) \leq(q+1) N_{1} \cdots N_{n}$. If $t \geq 5(q-p) ! l_{n}^{p-q}$, then $\beta\left(U_{p}, U_{q}, t\right) \geq N_{1} \cdots N_{n}$.

Proof: Upper bound of $\beta$ If for some subspace $L$ we have $t \cdot U_{q} \subset U_{p}+L$, then $\beta(t) \leq \operatorname{dim} L$. Let us fix $n$ such that $5 t \leq l_{n}^{p-q}$. Set $M=N_{1} \cdots N_{n}$. In the union $E_{n}=\bigcup_{k=1}^{M} I_{n, k}$ let $I_{n, k}=\left[a_{k}, b_{k}\right]$ briefly. For $k=1,2, \ldots, M$ and $j=0,1, \ldots, q$, let $e_{k, j}(x)=\frac{\left(x-a_{k}\right)^{j}}{j !}$ if $x \in K \cap I_{n, k}$ and $e_{k, j}(x)=0$ otherwise on $K$. We take $L=$ $\operatorname{Span}\left(e_{k, j}\right)_{k=1, j=0}^{M, q}$. Then $\operatorname{dim} L=(q+1) M$ and it is enough to show that for any function $f$ with $\|f\|_{q} \leq t$ there exists a function $g \in L$ such that $\|f-g\|_{p} \leq 1$. Given $f \in t U_{q}$ let $g=\sum_{k=1}^{M} \sum_{j=0}^{q} f^{(j)}\left(a_{k}\right) \cdot e_{k, j}$. Clearly, if $x \in I_{n, k}$, then $(f-g)(x)=$ $R_{a_{k}}^{q} f(x)$. Since $\left|x-a_{k}\right| \leq l_{n}$ we get

$$
\left|(f-g)^{(i)}(x)\right| \leq\|f\|_{q} \cdot\left|x-a_{k}\right|^{q-i} \leq t \cdot l_{n}^{q-i}, \quad i \leq p .
$$


Let now $A_{p}=\frac{\left|\left(R_{y}^{p}(f-g)\right)^{(i)}(x)\right|}{|x-y|^{p-i}}, x, y \in K, x \neq y, i \leq p$.

If $x, y \in I_{n, k}$ for some $k$, then as in [7, p. 569] we can use the following representation

$$
R_{y}^{p}(f-g)(x)=R_{y}^{q} f(x)+\sum_{m=p+1}^{q}\left(R_{a_{k}}^{q} f\right)^{(m)}(y) \cdot \frac{(x-y)^{m}}{m !}
$$

Therefore

$A_{p} \leq\|f\|_{q} \cdot|x-y|^{q-i} \cdot|x-y|^{i-p}+\sum_{m=p+1}^{q}\|f\|_{q} \cdot\left|y-a_{k}\right|^{q-m} \cdot \frac{|x-y|^{m-i}}{(m-i) !} \cdot|x-y|^{i-p}$.

Here $|x-y| \leq l_{n}$, hence $A_{p} \leq t \cdot l_{n}^{q-p}\left(1+\sum \frac{1}{(m-i) !}\right)<(e+1) t \cdot l_{n}^{q-p}$.

On the other hand, if the points $x, y$ are situated on different basic intervals of $E_{n}$, then $|x-y| \geq h_{n} \geq l_{n}$ by assumption. For this case

$$
A_{p} \leq\left|(f-g)^{(i)}(x)\right| \cdot|x-y|^{i-p}+\sum_{m=i}^{p} \frac{\left|(f-g)^{(m)}(y)\right|}{(m-i) !}|x-y|^{m-i+i-p} .
$$

From (4) it follows that $A_{p} \leq t \cdot l_{n}^{q-p}+t \cdot l_{n}^{q-p} \sum_{m=i}^{p} \frac{1}{(m-i) !}$. Thus, $\|f-g\|_{p} \leq$ $t \cdot l_{n}^{q-p}(2+e)<1$, by condition and this establishes the upper bound of $\beta$.

Lower bound of $\beta$ In order to find a lower estimate for Kolmogorov diameters we use the Tikhomirov theorem [20] (see also [11, Proposition 6]): if $d \cdot U_{p} \cap L \subset U_{q}$ with $\operatorname{dim} L=n+1$, then $d_{n}\left(U_{q}, U_{p}\right) \geq d$.

Therefore, $\beta(t) \geq \operatorname{dim} L$ if $U_{p} \cap L \subset\left(1-\epsilon_{0}\right) t \cdot U_{q}$ with some $\epsilon_{0}>0$. Let us take $L=\operatorname{Span}\left(e_{k, q}\right)_{k=1}^{M}$ and fix $f=\sum_{k=1}^{M} C_{k} \cdot e_{k, q} \in L \cap U_{p}$. Since $1 \geq\|f\|_{p} \geq$ $\left|f^{(p)}\left(b_{k}\right)\right| \geq\left|C_{k}\right| \frac{l_{n}^{q-p}}{(q-p) !}$, we have $\left|C_{k}\right| \leq(q-p) ! l_{n}^{p-q}$ for all $k$. Let $x \in I_{n, k}$. Then $\left|f^{(i)}(x)\right| \leq\left|C_{k}\right| \frac{l_{n}^{q-i}}{(q-i) !}, i \leq q$, hence $|f|_{q} \leq(q-p) ! l_{n}^{p-q}$.

If $x, y \in I_{n . k}$ then $R_{y}^{q} f(x)=0$. Otherwise, $|x-y| \geq h_{n} \geq l_{n}$ and arguing as before, we obtain

$$
\begin{aligned}
\left|\left(R_{y}^{q} f\right)^{(i)}(x)\right| \cdot|x-y|^{i-q} & \leq\left|f^{(i)}(x)\right| \cdot h_{n}^{i-q}+\sum_{m=i}^{q} \frac{f^{(m)}(y)}{(m-i) !} h_{n}^{m-q} \\
& \leq \frac{\left|C_{k}\right|}{(q-i) !}+\sum_{m=i}^{q} \frac{\left|C_{k_{1}}\right|}{(m-i) !} \leq(q-p) ! l_{n}^{p-q}(1+e) .
\end{aligned}
$$

If we take $\epsilon_{0}$ with $2+e<5\left(1-\epsilon_{0}\right)$, then $\|f\|_{q} \leq(q-p) ! l_{n}^{p-q}(2+e)<t \cdot\left(1-\epsilon_{0}\right)$ and $\beta(t) \geq \operatorname{dim} L=M$.

Now we can easily find the diametral dimension of $\mathcal{E}(K)$ for concrete compact set $K$. In particular for classical Cantor set we have $\beta\left(U_{p}, U_{q}, t\right) \sim t^{\frac{\log 2}{(q-p) \log 3}}$, that is the 
diametral dimension of $\mathcal{E}(K)$ is the same as $\Gamma(s)$. Here and subsequently, $F \sim G$ means that for some $C, t_{0}$ we have

$$
\frac{1}{C} F\left(\frac{t}{C}\right) \leq G(t) \leq C \cdot F(C t), \quad t>t_{0} .
$$

The most interesting case we have is for $K=K_{N}^{(\alpha)}$. Let $\lambda_{0}$ denote as before the logarithmic dimension of $K$.

Corollary 1 Let $X=\mathcal{E}\left(K_{N}^{(\alpha)}\right)$. Then $\beta\left(U_{p}, U_{q}, t\right) \sim \log ^{\lambda_{0}} t, t \rightarrow \infty$.

Corollary $2 \Gamma\left(\mathcal{E}\left(K_{N}^{(\alpha)}\right)\right)=\left\{\left(\gamma_{n}\right): \gamma_{n} \cdot \exp \left(-n^{\frac{1}{\lambda_{0}}}\right) \rightarrow 0\right.$ as $\left.n \rightarrow \infty\right\}$.

Corollary 3 If spaces of the type $\mathcal{E}\left(K_{N}^{(\alpha)}\right)$ are isomorphic, then the corresponding compact sets have the same logarithmic dimension.

Corollary 4 If $\alpha<N$, then the space $\mathcal{E}\left(K_{N}^{(\alpha)}\right)$ is isomorphic to a complemented subspace of $s$, but is not isomorphic to $s$.

Proof Corollary 1 is the result of a simple computation. Corollary 2 follows from Proposition 4, and Corollary 3 does so from Proposition 5.

By definition, $\mathcal{E}\left(K_{N}^{(\alpha)}\right)$ is a quotient space of $s$. If $\alpha<N$, then according to Theorem 1, the space $\mathcal{E}\left(K_{N}^{(\alpha)}\right)$ has the property DN. Thus, due to Vogt's characterization (see, e.g. [22]) it is a complemented subspace of $s$. But since $\Gamma\left(\mathcal{E}\left(K_{N}^{(\alpha)}\right)\right) \neq \Gamma(s)$, they are not isomorphic.

\section{Isomorphic Classification}

First we shall generalize Corollary 3 to Theorem 3 for compact sets of finite type.

Let $K_{N}=K\left(\left(l_{n}\right), N\right), K_{M}=K\left(\left(L_{m}\right), M\right)$. Without loss of generality we can take $l_{1}=L_{1}=1 / e$. Then $l_{n}=\exp \left(-\alpha_{1} \cdots \alpha_{n}\right), L_{m}=\exp \left(-\alpha_{1}^{\prime} \cdots \alpha_{m}^{\prime}\right)$.

Theorem 4 Let $X=\mathcal{E}\left(K_{N}\right), Y=\mathcal{E}\left(K_{M}\right)$. Suppose that the corresponding limits $\lambda_{X}=\lim _{n \rightarrow \infty} \frac{\log N}{\log \alpha_{n}}, \lambda_{Y}=\lim _{m \rightarrow \infty} \frac{\log M}{\log \alpha_{m}^{\prime}}$ exist in the set of extended real numbers and are not equal. Then the spaces $X$ and $Y$ are not isomorphic.

Proof To be definite, assume that $\lambda_{X}<\lambda_{Y}$. If $\lambda_{X}=0$, then $\alpha_{n} \rightarrow \infty$ and $\alpha_{n}^{\prime} \nrightarrow \infty$. By Theorem 1 in [8], it follows that the space $Y$ has the property $\underline{\mathrm{DN}}$ (for definition see e.g. [10]) whereas $X$ does not. So they are not isomorphic.

Consider now the case $0<\lambda_{X}<\lambda_{Y}<\infty$. Suppose, contrary to our claim, that $X \simeq Y$. Then by Proposition 4 , for $p_{1}=0$ there exists $p \in \mathbb{N}$ such that for $q=p+1$ one can find $q_{1} \in \mathbb{N}, \epsilon>0$ with

$$
\beta_{Y}\left(V_{0}, V_{q_{1}}, \epsilon t\right) \leq \beta_{X}\left(U_{p}, U_{p+1}, t\right), \quad t>0
$$


Assume $n$ and $m$ are large enough and are such that

$$
5 \cdot q_{1} ! L_{m}^{-q_{1}} \leq \frac{\epsilon}{5} l_{n}^{-1}
$$

Then by taking $t=\frac{1}{5} l_{n}^{-1}$ and using Theorem 3, we obtain

$$
M^{m} \leq \beta_{Y}\left(V_{0}, V_{q_{1}}, \epsilon t\right) \leq \beta_{X}\left(U_{p}, U_{p+1}, t\right) \leq(p+2) N^{n} .
$$

Set $\alpha=\lim \alpha_{n}, \alpha^{\prime}=\lim \alpha_{n}^{\prime}$. Clearly, $1<\alpha$, $\alpha^{\prime}<\infty$. Take $\rho>0$ with $\lambda_{X}=$ $(1-2 \rho) \lambda_{Y}$ and $\delta>0$ such that

$$
\frac{\log (\alpha-\delta)}{\log \left(\alpha^{\prime}+\delta\right)} \cdot \frac{\log \alpha^{\prime}}{\log \alpha}>1-\rho
$$

For this $\delta$ one can take $n_{0}, m_{0}$ such that $\left|\alpha_{n}-\alpha\right|<\delta, \log \frac{25 q_{1} !}{\epsilon} \leq \frac{\alpha_{1} \cdots \alpha_{n}}{2}$ for $n \geq n_{0}$ and $\left|\alpha_{m}^{\prime}-\alpha^{\prime}\right|<\delta, m \geq m_{0}$.

Let now

$$
\begin{gathered}
C_{1}=\frac{1}{2 q_{1}} \frac{\alpha_{1} \cdots \alpha_{n_{0}}}{\alpha_{1}^{\prime} \cdots \alpha_{m_{0}}^{\prime}} \frac{\left(\alpha^{\prime}+\delta\right)^{m_{0}}}{(\alpha-\delta)^{n_{0}}}, \quad C_{2}=\frac{\log C_{1}}{\log \left(\alpha^{\prime}+\delta\right)}, \\
m=m(n)=\left[n \cdot \frac{\log (\alpha-\delta)}{\log \left(\alpha^{\prime}+\delta\right)}+C_{2}\right] .
\end{gathered}
$$

Then

$$
\left(\alpha^{\prime}+\delta\right)^{m} \leq C_{1}(\alpha-\delta)^{n}
$$

and so for $n \geq n_{0}, m \geq m_{0}$ we get

$$
\log \frac{25 \cdot q_{1} !}{\epsilon}+q_{1} \cdot \alpha_{1}^{\prime} \cdots \alpha_{m}^{\prime} \leq \alpha_{1} \cdots \alpha_{n}
$$

This is equivalent to (5) and so we have

$$
M^{m} \leq(p+2) N^{n} \quad \text { or } \quad m \cdot \lambda_{Y} \cdot \log \alpha^{\prime} \leq \log (p+2)+n \cdot \lambda_{X} \cdot \log \alpha .
$$

But this contradicts the choice of $\delta$ and $m(n)$, as it is easy to check. Thus the spaces $X, Y$ are not isomorphic.

It remains to consider the case $\lambda_{m}^{\prime} \rightarrow \lambda_{Y}=\infty, 0<\lambda_{X}<\infty$. Since $\left(\alpha_{m}^{\prime}\right)^{\lambda_{m}^{\prime}}=M$ for all $m$, we see that there exists $m_{0}$ such that $\alpha_{m}^{\prime}<1+\frac{\log 2 M}{\lambda_{m}^{\prime}}$ for $m \geq m_{0}$. Let $\delta<\alpha-1$ and for $n \geq n_{0}$ let $\alpha_{n} \geq \alpha-\delta$. As before the inequality (6) implies the boundedness of $\frac{m}{n}$.

If now $\alpha_{1}^{\prime} \cdots \alpha_{m}^{\prime} \nrightarrow \infty$ as $m \rightarrow \infty$, then we have (6) for some $n_{1}$ and all $m$, a contradiction. Otherwise let $C_{3}=\frac{2 q_{1} \alpha_{1}^{\prime} \cdots \alpha_{m_{0}}^{\prime}}{\alpha_{1} \cdots \alpha_{n_{0}}} \cdot(\alpha-\delta)^{n_{0}}$. For $(6)$, it is enough to have

$$
C_{3} \cdot\left(1+\frac{\log 2 M}{\lambda_{m}^{\prime}}\right)^{m-m_{0}} \leq(\alpha-\delta)^{n}
$$


If now the sequence $\left(m / \lambda_{m}^{\prime}\right)$ is bounded, then $(7)$ is valid for an arbitrary $m$ and some fixed $n$ which is a contradiction. If $\varlimsup \frac{m}{\lambda_{m}^{\prime}}=\infty$, then we take large $n, m$ with $n-1<$ $\frac{2 \log 2 M}{\log (\alpha-\delta)} \cdot \frac{m-m_{0}}{\lambda_{m}^{\prime}} \leq n$. Here we have (7) and therefore (6) for some subsequences $\left(m_{j}\right)$, $\left(n_{j}\right)$ but the ratio $\frac{m_{j}}{n_{j}}$ together with $\lambda_{m_{j}}^{\prime}$ are unbounded. This contradiction completes the proof.

The situation with compact sets of infinite type is much more complicated. By similar arguments we can distinguish now the spaces corresponding to different types. Let $K_{\infty}=K\left(\left(L_{m}\right),\left(M_{m}\right)\right), M_{m} \nearrow \infty, L_{1}=1 / e$ and hence as before $L_{m}=\exp \left(-\alpha_{1}^{\prime} \cdots \alpha_{m}^{\prime}\right)$. Set $H_{m}=\frac{L_{m-1}-M_{m} \cdot L_{m}}{M_{m}-1}$.

Theorem 5 Let $X=\mathcal{E}\left(K_{N}^{(\alpha)}\right), Y=\mathcal{E}\left(K_{\infty}\right)$ and assume the limit $\lambda_{Y}=\lim _{m \rightarrow \infty} \frac{\log M_{m}}{\log \alpha_{m}^{\prime}}$ exists in the set of extended real numbers. Then the spaces $X$ and $Y$ are not isomorphic.

Proof We may assume that there exists a constant $C_{1} \geq 1$, such that $L_{m} \geq H_{m}^{C_{1}}, \forall m$. Otherwise by Theorem 3 in [8] the space $Y$ does not have the property $\underline{D N}$ and the result follows. Then we get

$$
L_{m-1}<2 M_{m} \cdot L_{m}^{\frac{1}{C_{1}}}
$$

and

$$
2 M_{m} \geq \exp \left(\alpha_{1}^{\prime} \cdots \alpha_{m-1}^{\prime}\left(\frac{\alpha_{m}^{\prime}}{C_{1}}-1\right)\right)
$$

Suppose that the spaces $X, Y$ are isomorphic. Arguing as above, we see that if $m$ and $n$ satisfy

$$
\log \frac{25 \cdot q_{1} !}{\epsilon}+q_{1} \cdot \alpha_{1}^{\prime} \cdots \alpha_{m}^{\prime} \leq \alpha^{n}
$$

then

$$
M_{1} \cdots M_{m} \leq(p+2) N^{n}
$$

Consider the case $\lambda_{Y}<\infty$. Here $\alpha_{m}^{\prime} \rightarrow \infty$, with $m \rightarrow \infty$ and $M_{m}<\left(\alpha_{m}^{\prime}\right)^{2 \lambda_{Y}}$ for large enough $m$. This contradicts (8).

Let now $\lambda_{Y}=\infty$. If $\alpha_{m}^{\prime} \rightarrow 1$, then we take $m=n$ in (9) and obtain a contradiction in (10) as $M_{m} \nearrow \infty$. It suffices to consider the case $\lambda_{Y}=\infty, \alpha_{m}^{\prime} \nrightarrow 1$. For large $m$ let us take $n=C_{2}+\frac{\log \left(\alpha_{1}^{\prime} \cdots \alpha_{m}^{\prime}\right)}{\log \alpha}$, where $C_{2}$ is fixed such that $n \in \mathbb{N}$ satisfies the condition (9).

From (10) we deduce that

$$
\left(\alpha_{1}^{\prime}\right)^{\lambda_{1}^{\prime}} \cdots\left(\alpha_{m}^{\prime}\right)^{\lambda_{m}^{\prime}} \leq(p+2) N^{C_{2}} \cdot\left(\alpha_{1}^{\prime} \cdots \alpha_{m}^{\prime}\right)^{\frac{\log N}{\log \alpha}}
$$

which is impossible as $\lambda_{m}^{\prime} \rightarrow \lambda_{Y}=\infty, \alpha_{m}^{\prime} \nrightarrow 1$.

Question Does the space $\mathcal{E}\left(K_{\infty}\right)$ have a basis? 


\section{References}

[1] C. Bessaga, A. Pelczyński and S. Rolewicz, On Diametral approximative dimension and linear homogeneity of F-spaces. Bull. Acad. Pol. Sci. 9(1961), 677-683.

[2] E. Bierstone, Extension of Whitney-Fields from Subanalytic Sets. Invent. Math. 46(1978), 277-300.

[3] K. Falconer, Fractal Geometry, Mathematical Foundations and Applications. John Wiley \& Sons, 1990.

[4] L. Frerick, Extension operators for spaces of infinitely differentiable Whitney functions. Habilitation thesis, 2001.

[5] A. Goncharov, A compact set without Markov's property but with an extension operator for $C^{\infty}$-functions. Studia Math. (1) 119(1996), 27-35.

[6] Perfect sets of finite class without the extension property. Studia Math. (2) 126(1997), $161-170$.

[7] A. Goncharov and M. Kocatepe, Isomorphic classification of the spaces of Whitney functions. Michigan Math. J. 44(1997), 555-577.

[8] A continuum of pairwise nonisomorphic spaces of Whitney functions on Cantor-type sets. Linear Topol. Spaces Complex Anal. 3(1997), 57-64.

[9] A. N. Kolmogorov, On the linear dimension of topological vector spaces. Dokl. Akad. Nauk SSSR 120(1958), 239-341 (Russian).

[10] R. Meise and D. Vogt, Introduction to functional analysis. Clarendon Press, Oxford, 1997.

[11] B. S. Mityagin, Approximate dimension and bases in nuclear spaces. Russian Math. Surveys (4) 16(1961), 59-127 (English translation).

[12] R. Nevanlinna, Analytic Functions. Springer-Verlag, Berlin-New York, 1970.

[13] W. Pawłucki and W. Pleśniak, Extension of $C^{\infty}$ functions from sets with polynomial cusps. Studia Math. 88(1988), 279-287.

[14] A. Pełczyński, On the approximation of S-spaces by finite dimensional spaces. Bull. Acad. Polon. Sci. 5(1957), 879-881.

[15] Ch. Pomerenke, Boundary Behavior of Conformal Maps. Springer-Verlag, Berlin-Heidelberg, 1992.

[16] E. M. Stein, Singular integrals and differentiability properties of functions. Princeton Univ. Press, 1970.

[17] M. Tidten, Fortsetzungen von $C^{\infty}$-Funktionen, welche auf einer abgeschlossenen Menge in $\mathbb{R}$ definiert sind. Manuscripta Math. 27(1979), 291-312.

[18] Kriterien für die Existenz von Ausdehnungoperatoren zu $\mathcal{E}(K)$ für kompakte Teilmengen $K$ von $\mathbb{R}$. Arch. Math. 40(1983), 73-81.

[19] - A geometric characterization for the property ( $\underline{\mathrm{DN}}$ ) of $\mathcal{E}(K)$ for arbitrary compact subsets $K$ of $\mathbb{R}$. Arch. Math. 77(2001), 247-252.

[20] V. M. Tikhomirov, On n-th diameters of compact sets. Dokl. Akad. Nauk SSSR (4) 130(1960), 734-737.

[21] D. Vogt, Characterisierung der Unterräume von (s). Math. Z. 155(1977), 109-117.

[22] D. Vogt and M. J. Wagner, Characterisierung der Quotienträume von s und eine Vermutung von Martineau. Studia Math. 67(1980), 225-240.

[23] V. P. Zahariuta, Some linear topological invariants and isomorphisms of tensor products of scale's centers. Izv. Severo-Kavkaz. Nauchn. Centra Vyssh. Shkoly 4(1974), 62-64 (in Russian).

Bilkent University

Department of Mathematics

06533 Bilkent

Ankara

Turkey

email: barslan@firstlinux.net goncha@fen.bilkent.edu.tr kocatepe@fen.bilkent.edu.tr 